\title{
Formal Analysis of the Communication of Probabilistic Knowledge
}

\author{
João Carlos Gluz ${ }^{12.3}$, Rosa M. Vicari ${ }^{1}$, Cecília Flores ${ }^{1}$, Louise Seixas ${ }^{1}$ \\ 1 Instituto de Informática, UFRGS,95501-970, Porto Alegre, RS, Brasil, \\ \{jgluz,rosa,dflores\}@infufrgs.br, seixasl@terra.com.br \\ 2 ESD, UERGS, Guaíba, RS, Brasil, joao-gluz@uergs.edu.br \\ 3 FACENSA, Gravatai, RS, Brasil, jcgluz@facensa.combr
}

\begin{abstract}
This paper discusses questions about communication of probabilistic knowledge in the light of current theories of agent communication. It will argue that there is a semantic gap between these theories and research areas related to probabilistic knowledge representation and communication, that creates very serious theoretical problems if agents that reason probabilistically try to use the communication framework provided by these theories. The paper proposes a new formal model, which generalizes current agent communication theories (at least the standard FIPA version of these theories) to handle probabilistic knowledge communication. We propose a new probabilistic logic as the basis for the model and new communication principles and communicative acts to support this kind of communication.
\end{abstract}

\section{Introduction}

This paper will present a theoretical study about which kind of meaning can be assigned to the communication of probabilistic knowledge between agents in Multiagent Systems (MAS), at least when current theories for agent communication are considered. The work starts in section 2, presenting several considerations showing that exists a semantic gap between current agent communication theories and research areas related to probabilistic knowledge representation and communication. This gap creates very serious theoretical problems if the designer of agents that reason probabilistically tries to use the communication framework provided by these theories to model and implement all agent's communication tasks.

To minimize this gap we propose a new formal model in section 3 , which generalizes the formal model, used in FIPA agent communication standards [6], to handle probabilistic knowledge communication. We propose a new probabilistic logic, called $S L P$, as the basis for the new model. The SLP logic is compatible with the logic used as the foundation of FIPA standards (the $S L$ logic) in the sense that all valid formulas (theories) of $S L$ are also valid formulas of $S L P$. The axiomatic system of $S L P$ is correct. It is also complete, if the axiomatic system of $S L$ is complete.

Please use the following format when citing this chapter:

Gluz, J.C., Vicari, R.M., Flores, C., Seixas, L., 2006, in IFIP International Federation for Information Processing, Volume 217 , Artificial Intelligence in Theory and Practice, ed. M. Bramer, (Boston: Springer), pp. 31-40. 
Based on SLP logic we propose a minimum set of new communication principles in section 4 that are able to correlate probabilistic reasoning with communication related inference tasks. Two new communicative acts are proposed that would allow agents to communicate basic probabilistic propositions without having to agree previously on a probabilistic content format.

This is the most important result of the paper. To our knowledge, this is the first work that tries to integrate in a single probabilistic-logical framework two entirely different approaches to understand and model communication. What we have done, after have carefully isolated formal axiomatic agency and communication theories used by FIPA, was to define the minimum set of new axioms necessary and sufficient to support an probabilistic form of assertive and query communicative acts. We also maintain the principles, acts and axioms as simple as possible to be able to easily assess how much we were departing from classical Speech Act theory. We believe, that given the circumstances, albeit a conservative approach, this is the correct approach. The result was a clear and simple generalization of current FIPA axiomatic communication and agent theories that is able to handle basic probabilistic communication between agents.

A secondary, but interesting, result of the paper is the (relative) completeness of SLP logic. To our knowledge, there is no other axiomatization for an epistemic and temporal modal logic, which allow probabilities for first order modal sentences, and is proved complete.

\section{Motivation}

This work has started with a very practical and concrete problem, which was how to model (and implement) the communication tasks of all agents from a real MAS: the AMPLIA system [13,8]. We have decided to use only standard languages and protocols to model and implement these tasks in order to allow reusability of the agent's knowledge and to allow an easier interoperation of AMPLIA with others intelligent learning systems. To this purpose we decided to use FIPA standards based on two assumptions: (a) the standards are a good way to ensure MAS knowledge reusability and interoperability; (b) the formal basis of FIPA standards offer an abstract and architecture independent way to model all communication tasks of the system, allowing a high level description of the communication phenomena. However, we have found that it was impossible to meet even most basic communication requirements of AMPLIA using only FIPA standards. All AMPLIA's agents use and communicate probabilistic (bayesian) knowledge, but FIPA standards assigns no meaning to probabilistic knowledge representation or communication.

Of course it is possible to try to "hide" all probabilistic knowledge in a special new content format, allowing, for example, that Bayesian Networks (BN) should be "encoded" in this format and then embedded as contents of FIPA Agent Communication Language (ACL) communicative acts. The knowledge to be passed as contents of assertive acts like FIPA's inform, can be considered as a logical proposition that the agent believe it is true. In being so, it is possible to assume that, from a communication point of view, it is only necessary that the agent believe that the "hidden" probabilistic knowledge transported by the act be true. Any other meaning related the probabilistic knowledge do not need be "known" by the agent in respect to communication tasks or in any reasoning related to these tasks. 


\subsection{The Research Problem}

The approach to "hide" probabilistic knowledge solves some basic implementation problems if theoretical or formal aspects of this kind of communication are not considered. However, when analyzed more carefully this approach does not seem to be very sound.

The first problem is related to the fact that formal semantics of FIPA ACL is based on axiomatic logical theories of intention and communication $[4,5,11,12]$. Besides particular pre and pos-conditions (expressed as logical axioms) for some act, these theories will define clearly when the act should be emitted, what are the intentions of the sender agents when it send the act, which effects this act should cause in the receiver agent and so on. The knowledge transported in these acts are only logical propositions, but these propositions are related to internal beliefs, intentions and choices of the agents and must be used in reasoning process that will decides when to emit some act or how the act received should be understood. This imply that even if you have some probabilistic knowledge "hidden" in the contents of a communicative act, then this knowledge cannot be used in any internal reasoning process related to communication tasks, because formal model and theories that fundament this reasoning (at least in FIPA standards) are purely logical and do not allow reasoning about probabilities. This generates a strange situation when you have an agent with probabilistic reasoning abilities: the agent can "think" probabilistic in all internal reasoning, but never can "think" probabilistically when talking, listening and trying to understand (i.c. communicating) other agents, at least when purely logical theories are used to fundament the communication. It has the additional consequence that an agent that reason only by probabilistic means cannot "use" FIPA acts, languages and protocols if it wants to keep theoretical consistency.

The second question arises from epistemological and linguistic considerations, when we take into account agents that can reason probabilistically. We will assume that the agent uses subjective (bayesian) reasoning and can assign probabilities to his beliefs, that is, the agent can reason with degrees of belief. Assuming only basic rationality for this kind of agent, then, if it has some probabilistic belief and needs to inform this belief to another agent it will need to be sure that the proper degree of belief be also correctly informed. For instance, if it strongly believes ( $90 \%$ of chance) that it will rain tomorrow and need to inform this belief to another agent to change his behavior (for example, to cancel some encounter), then it will need to convince the other agent to have the same strong belief about the possibility to rain tomorrow. Some appropriate locus for the transportation of this kind of probability needs to be found in current theories of communication. The problem is that the Speech Act Theory of Searle and Grice, which provides the epistemological and linguistic basis for formal communication theories, simply do not consider the possibility of agents to communicate knowledge of probabilistic nature because the most basic semantic "unit" of knowledge that is considered by the theory is a logical proposition. Consequently, all formal theories of communication (including, the Theory of Action, Intention and Communication of Cohen, Levesque [4,5] and Sadek [11,12]) have adopted this point of view and do not consider probabilistic knowledge communication as a real possibility.

Together both questions create a very interesting dilemma: if an agent use probabilistic reasoning and need to inform some probabilistic belief to another agent it will have serious problems to do this task, because current linguistic theories say that there is no means to accomplish it (according to these theories there is no locus to communicate probabilities). These theories, at least in their formal counterpart, say even more, stating that even if you can send this probabilistic knowledge there is no way to consider this knowledge when reasoning 
about communication tasks. This surely is not a good situation from a theoretical point of view, and our work will try to start to correct this problem, at least in the limited sense of FIPA formal agent communication model.

\subsection{Related Work}

The problems expressed in previous sub-section are not addressed in recent research literature about ACLs (see [3]). Research in this area and related areas of agent societies and social interaction is more focused in the study about logical aspects of social institutions, including trust relationship, intentional semantics for social interaction and similar concepts, but not in checking the role of probabilities in these concepts. A similar situation also occurs in the research area of probabilistic knowledge representation for MAS. Main papers in those areas are focused on the question of how to communicate and distribute BN probabilistic knowledge between agents [14], keeping the inference processes consistent, efficient and epistemologically sound. These pieces of research offer a separate form of knowledge representation and communication not related to ACL research. Our work intends to start to bridge this gap, by showing how probabilistic knowledge can be included in the FIPA communication framework in an integrated and uniform way.

Our approach to formalize the communication of probabilistic knowledge is based on the idea that the best way to do this, in a way that is integrated and compatible with current agent communication theories (at least in the FIPA case), is to use a modal logic that can handle probabilities, that is, to use a probabilistic logic. In terms of Artificial Intelligence research, probabilistic logics were first described by Nilsson [10], already using a possible-worlds model to define the semantic of his logic. The initial work of Nilsson was profoundly extended, in the beginnings of 1990, by the works of Halpern [9], Abadi [1] and Bacchus [2] mainly related to epistemic (or doxastic) probabilistic modal logics. Currently there is also an active line of research based on probabilistic extensions to the $\mathrm{CTL}^{*}$ temporal logic from Emerson and Srinavan, like the PCTL logic of Segala. However, due to the nature of the theories of agent communication, that require BDI modal operators, we focused our research only on epistemic probabilistic modal logics.

\section{SLP Probabilistic Logic}

\subsection{FIPA's SL Logic}

The SL (Semantic Language) is a BDI-like modal logic with equality that fundaments FIPA communication standards. This logic was defined by Sadek's work [11,12], which attributes a model-based semantics for $S L$ logic. In $S L$, there is no means of attributing any subjective probability (or degree of belief) to a particular belief of some agent, so it is not possible to represent or reason about probabilistic knowledge in this logic.

Besides the usual operators and quantifiers of the predicate logic with equality, $S L$ contains modal operators to express the beliefs $(\boldsymbol{B}(a, \varphi))$, choices $\boldsymbol{C}(a, \varphi)$ and intentions $(\boldsymbol{I}(a, \varphi))$ of an agent $a$. $S L$ also has a relatively obscure modal operator that defines an "absolute uncertainty" that an agent can have about some belief. The $U(a, \varphi)$ operator, however, does not admit any kind of degree or uncertainty level. There is no clear connection between probability theory and $U$ operator. It is also possible to build action expressions that can be connected in series $e_{1} ; e_{2} ; \ldots ; e_{\mathrm{n}}$, in altemates $e_{1} \mid e_{2}$ or verified by an agent $a(a, e)$ ?. Temporal and possibil- 
ity assertions can be made based on the fact that an action or event has happened (Donete, $\varphi)$ ), on the possibility that an action or event may happen (Feasible(e, $\varphi)$ ) and on which agent is responsible for an action (Agent $(a, e, \varphi))$.

\subsection{The SLPLogic}

The extension of the $S L$ logic is called SLP, for Semantic Language with Probabilities, and it is defined through the extension of the $S L$ formal model. For such purpose, $S L P$ will incorporate numerical operator, relations and expressions, and terms that denote probabilities expressing the subjective probability (degree of belief) of a given sentence or statement being true.

The probabilistic term $B P(a, \varphi)$ is specific for $S L P$ and informs the probability of a proposition $\varphi$ be true with respect to the beliefs of agent $a$, that is, it defines the subjective probability assigned to $\varphi$ by $a$. For example, $B P(a, \exists(x)(\mathrm{P}(x)) \leq 1$ express the fact that the subjective probability assigned by agent $a$ to the possibility that some element of the domain satisfies $\mathrm{P}(x)$ is less than 1 .

The model-based semantics for formulas of SLP is defined over a set $\Phi$ of symbols for variables, functions, predicates, primitive actions, agents and constants through models $M$ with the following structure:

$$
M=<\mathcal{W}, A g t, \mathcal{E} w t, \text { Obj, } B, C, E, A G \mathcal{T}, \sigma, R C F, \mu>
$$

The elements $\mathcal{W}, A g t, O 6 j, \mathcal{E} v t, B, C, \mathcal{E}, \mathcal{A} G T$ and $\sigma$ are part of the formal model originally defined for $S L$ by Sadek [12]. They define the set of possible worlds ( $\mathcal{W}$ ), agents (Agt), primitive events (Evt), objects $(O 6 j)$ and causative agent for primitive events $(A G T)$ of $S L P$. They also define the set of accessibility relations for beliefs $(B)$, choices $(C)$ and future worlds $(\mathscr{E})$ of SLP. The mapping $\sigma$ denotes a standard first-order logic interpretation that attributes, for each possible world, function and predicate symbol in $\Phi$ a correspondent element in $A g t \cup$ $O 6 j \cup$ Evt (the logical domain of SLP).

The elements $\mu$ and $R C F$ are new elements specifically defined to SLP. The set $\mu$ is a set of mappings that attributes to each agent $a$ a discrete probability distribution function $\mu_{a}$ on the set of possible-worlds $\mathcal{W}$. The basic restriction to this set of mappings is that any mapping $\mu_{a}$ must respect the restrictions for any discrete probability function. The symbol $R C F$ denotes the (up to isomorphism) closed field of real numbers. RCF it is the domain for the purely numerical formulas of SLP and includes addition and multiplication operations on real numbers, the neutral elements of these operations, the partial ordering $\leq_{\mathrm{rcf}}$ and it satisfies all properties of real closed fields.

The formal semantics of $S L P$ expressions, that are not probabilistic, are identical to the semantics given for $S L$ in [12]. The presentation of the semantic for the entire $S L P$ logics is out of the scope of present work (it is defined in [7]), however, here we will define the formal semantics of the basic belief relation $\boldsymbol{B}(a, \varphi)$ and of the new probabilistic term $\boldsymbol{B P}(a, \varphi)$, to show the correlation between these two constructions.

Definition 1. The modal operator $\boldsymbol{B}(a, \varphi)$ expresses the fact that the agent a beliefs that the sentence $\varphi$ is true in a model $M$, world $w$ and evaluation function $v$ if and only if $\varphi$ is tmie in any world $w$ ' which can be reached from $w$ using ${ }^{B}{ }_{a}$ the belief accessibility relation for the agent a: 


$$
M, w, v \models B(a, \varphi) \text { iff } M, w^{\prime}, v \models \varphi, \quad \text { for all } w^{\prime} \text { such that } w B_{a} w^{\prime} .
$$

Definition 2. The semantic of the probabilistic term BP( $a, \varphi)$ is the probability estimated by agent $a$ that $\varphi$ is true. This probability is calculated summing up the distribution function $\mu_{a}$ over the worlds where agent a believe that $\varphi$ is true:

$$
\left[\left.\boldsymbol{B P}(a, \varphi)\right|_{M, w, v}=\mu_{a}\left(\left\{w^{\prime} \mid w B a^{w} w^{\prime} \text { and } M, w^{\prime}, v \models \varphi\right\}\right)\right.
$$

Besides these definitions, we add two assumptions to the formal model of SLP.

Assumption 3. The following equivalences are valid in SLP:

$B(a, \varphi) \Leftrightarrow B P(a, \varphi)=1$

$$
U(a, \varphi) \Leftrightarrow B P(a, \varphi)=0.5
$$

This assumption states the basic relationship between probabilistic and non-probabilistic (i.e. purely logical) beliefs in $S L P$ and between "absolute" uncertainties and probabilistic beliefs.

Assumption 4. Any formula $\varphi$ inside BP(a, $\varphi)$ terms must be a sentence (a closed formula) of the logic. Numerical constants or variables cannot be used as arguments of logical predicates (and vice-versa).

The axiomatic system of SLP was built over the axiomatic system of $S L$. It incorporates all axioms and inference rule from $S L$. To support probabilities were added the axiomatic system for the real closed field of numbers and axioms and inference rules equivalent to Kolmogorov axioms for Probability Theory.

\subsection{Properties of SLP Logic}

The basic properties of SLP are enunciated in the following propositions.

Proposition 5. Any valid formula of SL is also a valid formula of SLP and any purely logical valid formula of SLP is a valid formula of SL. $\square$

The proof of this proposition is not so simple because of assumption 3 which forces that every world with nonzero probability from a $M$ model can be reached by any other world of this model through the $B$ relation, something that is not required in $S L$ (or in other epistemic modal logics). Even so, it was possible to prove in [7], that any valid model of $S L$ is also a valid model of $S L P$ and vice-vers $a$ and thus proves the proposition 5.

Proposition 6. The axiomatic system of SLP is correct. $\square$

The new axioms and inference rules of $S L P$ are derived from the axiomatic theory of probabilities from Kolmogorov and from the axiomatic theory of the real field, both proved correct axiomatic systems.

In our proposed extension to $S L$, we have taken special care to avoid the problem of undecidability of probabilistic logics described in [1]. We have found a very interesting result, showing that there is a simpler and intuitive set of restrictions, not so strong as the restrictions proposed by Halpern and Bacchus that keep the resulting axiomatic system complete.

Proposition 7. The axiomatic system of SLP is complete if the axiomatic system of SL is also complete. $\square$

The basic insight that lead us to the (relative) completeness proof of SLP was based on the observation that the incompleteness proof for probabilistic logics made by Abadi and Halpern [1] relied on the fact that the same variables can be "shared" by terms inside probabilistic operator and logical formulas outside these operators, i.e., it is possible to have expressions like $P(x, y) \wedge \boldsymbol{B P}(Q(x))=r$, where the variable $x$ is shared by $P(x, y)$ and $Q(x)$ inside the $\boldsymbol{B P}$ operator. The consequence is that if we not allow shared variables between probabilistic terms and logical formulas, then Abadi technique will not work. This is not the 
istic terms and logical formulas, then Abadi technique will not work. This is not the same to say that the corresponding axiomatic system is complete, but it shows that this should be possible. Indeed, if we do not allow this kind of sharing, as is the case of SLP because of assumption 4, it is possible to use proof techniques developed by Halpern [9] and separate the probabilistic and non-probabilistic parts of some formula. This is the basic method employed on the completeness proof of SLP. In [7] it was shown that the validity of any formula $\varphi$ of $S L P$ can be reduced to the validity of an equivalent formula $\psi \wedge \pi$, where $\psi$ is a purely logical formula containing no numerical or probabilistic term and $\pi$ is a purely numerical formula containing no logical predicate/term neither any probabilistic term.

In this case, the validity of formula $\psi$ is entirely dependant on the original $S L$ axiomatic system and the validity of $\pi$ depends on the first order axiomatic theory of real closed fields that, by a well-known result of Tarski, is a decidable problem. This result was proved using a finitary generalization of the Halpern techniques presented in [9] to substitute probabilistic terms that contain closed first order modal formulas with universally quantified numerical variables.

\section{Communication of Probabilistic Knowledge}

\subsection{Principles for Probabilistic Communication}

The FIPA ACL semantic depends on several logical axioms that define principles for agency and communication theories (see $[11,12]$ for details). The theory of agency employed by FIPA includes rationality, persistency and consistency principles for beliefs, choices and intentions of agents defined as $S L$ axioms and theorems. The theory of communication is formed by several axioms that define communication principles like the belief adjustment, sincerity, pertinence and cooperation principles besides the 5 basic communication properties stated in FIPA ACL specification [6]. These principles are generally sufficient to handle reasoning needs for communication purposes in any rational $\mathrm{BDI}$ agent that is FIPA compliant (at least when the sender's agent centered semantics used by FIPA ACL is appropriate for the application or domain in question). In being so, our first principle can be stated as the following assumption.

Assumption 8 Agents that need to communicate probabilistic knowledge and intend to use FIPA-ACL should also respect the theory of agency and the theory of communication proposed in FIPA standards.

This assumption is perfectly reasonable because of compatibility between $S L$ and $S L P$ assured by proposition 5, that implies that any valid theory of $S L$ is a valid theory of $S L P$. However, when agents use probabilistic reasoning and need to use this kind of knowledge for communication purposes, then the purely logical theories of agency and communication are not much useful. To handle these situations we propose that these theories be extended by two new principles that will be able to bridge the gap between purely logical considerations and probabilistic reasoning, in terms of agent's communication decisions. We will propose only a minimum set of new principles, strictly necessary to correlate probabilistic knowledge used by the agent to decision and inference processes related to communication tasks.

One fundamental property of FIPA theory is the principle that assures the agreement between the mental state of some agent and their beliefs [12]. Using this principle is possible to assert propositions like $\boldsymbol{B}(a, \varphi) \leftrightarrow \boldsymbol{B}(a, \boldsymbol{B}(a, \varphi))$ and $\boldsymbol{B} \boldsymbol{P}(a, \varphi)=\mathbf{1} \leftrightarrow \boldsymbol{B}(a, \boldsymbol{B P}(a, \varphi)=\mathbf{1})$, if all 
propositions and predicate symbols in $\varphi$ appears in the scope of a modal operator formalizing a mental attitude of agent $a$ :

This is an interesting fact but is very limited in the case of probabilistic communication. The principles of FIPA's theory of communication assume that the agent must believe nonprobabilistically in some fact, before the communication starts. Therefore, what we need is some principle that will allow us to correlate probabilistic beliefs with non-probabilistic beliefs. This is assured by the following proposition of $S L P$.

Proposition 9. Principle of Probabilities and Beliefs Agreement: if some agent a assume that the probability of proposition $\varphi$ is $p$, then this is equivalent to state that it also believe in this fact:

$$
=\boldsymbol{B} \boldsymbol{P}(a, \varphi)=p \leftrightarrow \boldsymbol{B}(\boldsymbol{a}, \boldsymbol{B P}(a, \varphi)=p) \square
$$

This principle allows agents to put any probabilistic beliefs "inside" epistemic belief operators and then to use any other axioms and theorems of communication or agency theories to make communication related reasoning.

The proposition 9 is necessary but is not enough. We need some kind of reason to effectively start some new communicative act. In FIPA this is assured by the principle of belief adjustment [12] that states that if some agent $a$ believe in $\varphi$, believe that is competent in this belief and thinks that another agent $b$ do not believe in $\varphi$, then it adopts the intention to make $b$ believe in $\varphi$.

$$
\models \boldsymbol{B}(a, \varphi \wedge \boldsymbol{B}(b, \neg \varphi) \wedge \operatorname{Comp}(a, \varphi)) \rightarrow \boldsymbol{I}(a, \boldsymbol{B}(b, \varphi))
$$

The predicate $\operatorname{Comp}(a, \phi)$ states the competence of agent $a$ about $\varphi$.

The belief adjustment principle also falls in the same limiting situation of the mental state and belief agreement principle when applied to the probabilistic case. Therefore, we need another principle stated in the following proposition.

Proposition 10. Principle of Probabilities Adjustment: if some agent a believe that the probability of proposition $\varphi$ is $p$, believe that it is competent in this belief and also believe that another agent $b$ have different estimation for the probability of $\varphi$, then it should adopt the intention to make agent $b$ also believe that the probability of $\varphi$ is $p$ :

$\models \boldsymbol{B P}(a, \varphi)=p \wedge \boldsymbol{B} \boldsymbol{P}(a, \boldsymbol{B} \boldsymbol{P}(b, \varphi)=p)<\mathbf{1} \wedge \boldsymbol{B}(a, \operatorname{Comp}(a, \boldsymbol{B P}(a, \varphi)=p))) \rightarrow \boldsymbol{I}(a, \boldsymbol{B} \boldsymbol{P}(b, \varphi)=p) \square$

This principle is derived from belief adjustment principle, using the proposition 9 stated before (see [7] for details). It will have the same function of belief adjustment principle for the probabilistic reasoning case, providing agents with intentions to solve perceived differences between probabilistic beliefs shared by several agents.

\subsection{Communicative Acts for Probabilities}

Like $S L, S L P$ also can be used as a content representation language for FIPA-ACL communicative acts. This allows the representation and distribution of probabilistic knowledge like BN between agents using standard assertive (inform) acts. However, to do this is necessary to assume a particular structure in the contents of these acts. The assertive acts defined in Speech Act theory (and the equivalent inform FIPA-ACL acts) do not assume any particular internal structure in the propositions passed as contents of these acts. So, in the general case of probabilistic communication not seem reasonable to always assume a particular structure in the content of assertive act used to communicate probabilities. To handle this we propose that the strength (or weakness) of the assertive force of some speech act should be measured by a probability. In this way, any kind of propositions can be used as contents of these probabilistic assertive acts, because the (subjective) probability of the proposition will 
be transmitted as a graduation of the force. This graduation is a numerical coefficient that represents the subjective probability of the proposition (i. e., the graduation of the assertive force is directly related to the belief degree on the proposition). Two new probabilistic communicative acts were defined. They are considered extensions to the FIPA-ACL, creating the Probabilistic Agent Communication Language (PACL).

The acts inform-bp and query-bp acts are defined, respectively, to allow that the information about subjective probabilities of an agent to be shared with other agents and to allow that a given agent could query the degree of belief of another agent. Using the notation employed by FIPA-ACL [6] the inform-bp act is formalized as follows:

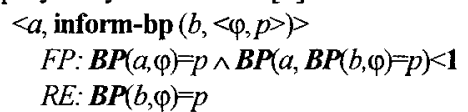

This act informs the probability for some closed formula $\varphi$. The feasibility precondition of the act $(F P)$ requires only that an agent to believe that the subjective probability of $\varphi$ is $p$ and that another agent $b$ has the chance of not believing in this fact. In this case, if the other necessary conditions are fulfilled (see [6]), then the inform-bp act will be emitted. The rational effect $(R E)$ that is expected with the act emission is that agent $b$ also comes to believe that the probability of $\varphi$ is $p$.

The query-bp act was also modeled after an analysis of the query-if act, which is its similar when dealing with truth-values. This directive act is used to retrieve the probabilistic information associated to a particular proposition.

\subsection{Examples}

The use of inform-bp acts is straightforward. Assume that some agent $a$ believe that agent $b$ have a different estimation of the probability of $\varphi$ and also believe that his estimation is competent:

$$
\boldsymbol{B} \boldsymbol{P}(a, \varphi)=p \wedge \boldsymbol{B}(a, \boldsymbol{B P}(b, \varphi) \neq \boldsymbol{B} \boldsymbol{P}(a, \varphi)) \wedge \boldsymbol{B}(a, \operatorname{Comp}(a, \boldsymbol{B P}(a, \varphi)=p)))
$$

Using the axioms and inference rules of $S L P$ it is possible to infer, from $B(a$, $\boldsymbol{B P}(b, \varphi) \neq \boldsymbol{B} \boldsymbol{P}(a, \varphi))$ and $\boldsymbol{B} \boldsymbol{P}(a, \varphi)=p$, that $-\boldsymbol{B}(a, \boldsymbol{B} \boldsymbol{P}(b, \varphi)=p)$. But this is equivalent to $\boldsymbol{B P}(a, \boldsymbol{B P}(b, \varphi)=p)<\mathbf{1}$, resulting:

$\boldsymbol{B P}(a, \varphi)=p \wedge \boldsymbol{B P}(a, \boldsymbol{B P}(b, \varphi)=p)<\mathbf{1} \wedge \boldsymbol{B}(a, \operatorname{Comp}(a, \boldsymbol{B P}(a, \varphi)=p)))$

Then, by (2) and proposition 10 the agent $a$ need to assume the intention to inform $b$ about the probability of $\varphi$. By the communication theory of FIPA this intention and beliefs stated in (2) are enough to cause the emission of the inform-bp act from $a$ to $b$ agent informing the probability of $\varphi$.

If we force that agents $a$ and $b$ use $S L P$ as content language and require that agent $a$ be completely unsure if agent $b$ knows the probability of $\varphi$, then it is also possible to use the inform acts of FIPA. The principle stated in proposition 9 allows to infer, from $\boldsymbol{B P}(a, \varphi)=p$, that:

$$
\boldsymbol{B}(a, \boldsymbol{B P}(a, \varphi)=p) \text {. }
$$

In FIPA inform act, the feasibility precondition $(F P)$ also requires that agent $a$ be completely unsure if the agent $b$ knows some proposition $\psi$ is stated as:

$\neg \boldsymbol{B}(a, \boldsymbol{B}(b, \psi) \vee \boldsymbol{B}(b, \neg \psi) \vee \boldsymbol{U}(b, \psi) \vee \boldsymbol{U}(b, \neg \psi))$

Substituting $\psi$ in (4) by $\boldsymbol{B} \boldsymbol{P}(b, \varphi)=p$ we have:

$$
\neg \boldsymbol{B}(a, \boldsymbol{B}(b, \boldsymbol{B} P(b, \varphi)=p) \vee \boldsymbol{B}(b,-\boldsymbol{B} \boldsymbol{P}(b, \varphi)=p) \vee \boldsymbol{U}(b, \boldsymbol{B} \boldsymbol{P}(b, \varphi)=p) \vee \boldsymbol{U}(b, \neg \boldsymbol{B} \boldsymbol{P}(b, \varphi)=p))
$$


So, agent $a$ believes in (3) and if it also believes in (5) it can emit an inform act to agent $b$, with the proposition $B P(a, \varphi)=p$ as the content of the act.

\section{Future Works}

Several interesting developments can follow our work. A direct possibility it is to check the influence of probabilistic knowledge and reasoning in other types of communicative acts and interaction protocols. Particularly interesting and related to our ongoing research it is the application of probabilistic knowledge and reasoning to model formally negotiation protocols, mainly when these protocols are related to the pedagogical negotiation, which is a very complex form of interaction that occurs in intelligent learning environments (and classrooms) [8]. Another possibility is to use the logical representation schemes for BN (like the schemes presented in [2] and [7]) as a starting point for the research of shared ontologies for probabilistic knowledge. The considerable research work already done for logical based ontologies, can be applied to this new research.

\section{Acknowledgments}

The authors gratefully acknowledge the Brazilian agencies CAPES, CNPq and FAPERGS for the partial support to this research project

\section{References}

[1] M. Abadi and J. Halpern. Decidability and Expressiveness for First-Order Logics of Probability. In Procs of IEEE Symp. on Foundations of Computer Science, 30, 1989.

[2] F. Bacchus. Lp, a Logic for Representing and Reasoning with Statistical Knowledge. Computational Intelligence, 6:209-301, 1990.

[3] B. Chaib-Draa and F. Dignun. Trends in Agent Communication Language. Computational Intelligence v. 2, n. 5. Cambridge, MA: Blackwell Publ, 2002

[4] P. Cohen and H. Levesque. Rational Interaction as the Basis for Communication, In P. Cohen, J. Morgan and M. Pollack (Ed.). Intentions in Communication. Cambridge, MA: MIT Press, 1990.

[5] P. Cohen and H. Levesque. Communicative Actions for Artifical Agents. In Procs of ICMAS-95. San Francisco. Cambridge: MIT Press, 1995.

[6] FIPA. FIPA Communicative Act Library Specification, Std. SC00037J, FIPA, 2002.

[7] J. C. Gluz. Formalization of the Communication of Probabilistic Knowledge in Multiagent Systems: an approach based on Probabilistic Logic (In Portuguese). PhD Thesis. Instituto de Informática, UFRGS, Porto Alegre, 2005.

[8] J. C. Gluz, C. Flores, L. Seixas and R. Viccari, R. Formal Aspects of Pedagogical Negotiation in AMPLIA System. In: Procs of T7SE-2005. Santiago, Chile, 2005.

[9] J. Y. Halpem. An Analysis of First-Order Logics of Probability. Antificial Intelligence, 46: 311-350, 1990.

[10] N. J. Nilsson. Probabilistic Logic. Artificial Intelligence, Amsterdan, 28: 71-87, 1986.

[11] M. D. Sadek. Dialogue Acts are Rational Plans. In: Procs. of ESCA/ETRW Workshop on the Structure of Multimodal Dialogue, Maratea, Italy, 1991.

[12] M. D. Sadek. A Study in the Logic of Intention.' In: Procs. of KR'92, p. 462-473, Cambridge, USA, 1992.

[13] R. M. Vicari, C. D. Flores, L. Seixas, A. Silvestre, M. Ladeira, H. A. Coelho. Multi-Agent Intelligent Environment for Medical Knowledge. Artificial Intelligence in Medicine, 27(3): 335-366, March 2003.

[14] Y. Xiang, A probabilistic framework for cooperative multi-agent distributed interpretation and optimization of communication. Artificial Intelligence, 87: 295-342, 1996 